\title{
RINGS WITH IDEMPOTENTS IN THEIR NUCLEI
}

\author{
BY
}

\section{MICHAEL RICH}

ABSTRACT. Let $R$ be a prime nonassociative ring. If the set of idempotents of $R$ is a subset of the nucleus of $R$ or of the alternative nucleus of $R$ then it is shown that $R$ is respectively an associative or an alternative ring. Also if $R$ has one idempotent $\neq 0,1$ which is in the Jordan nucleus or in the noncommutative Jordan nucleus then it is shown that $R$ is respectively a Jordan or a noncommutative Jordan ring.

Introduction. The purpose of this paper is to demonstrate that the degree of associativity of a prime, not necessarily associative ring can be determined from the associativity or lack thereof of the idempotents. Throughout we assume that the ring contains at least one idempotent $\neq 0,1$. We consider four cases. First, it is easily shown that if $R$ is a prime ring all of whose idempotents lie in the nucleus then $R$ is associative. This motivates consideration of the case in which all of the idempotents lie in an appropriate alternative nucleus of the ring. Similarly, the result here is that the ring is alternative. We next consider a prime commutative ring in which at least one idempotent $\neq 0,1$ lies in an appropriate Jordan nucleus and show that this implies that the ring is a Jordan ring. Finally, we consider prime flexible rings with at least one idempotent in the appropriate noncommutative Jordan nucleus with the result being that the ring is a noncommutative Jordan ring. Examples are given to show that the conditions assumed are necessary. The latter two cases generalize a result of Osborn.

As usual, the associator $(x, y, z)$ denotes $(x y) z-x(y z)$ and the commutator $[x, y]=x y-y x$. Also $R^{+}$is the same additive group as $R$, but multiplication in $R^{+}$is given by $a \cdot b=1 / 2(a b+b a), a b$ being the multiplication in $R$. Of course, this is meaningful only if $1 / 2 a$ is meaningful for all $a$ in $R$. A ring is called flexible if $(x, y, x)=0$, alternative if $(y, x, x)=(x, x, y)=0$,

Received by the editors January 17, 1974. 16 A32.

AMS (MOS) subject classifications (1970). Primary 17D05, 17C99, 17A15,

Key words and phrases. Alternative nucleus, Jordan nucleus, noncommutative Jordan nucleus, flexible, idempotent, prime ring. 
Jordan if $[x, y]=\left(x^{2}, y, x\right)=0$ and noncommutative Jordan if it is flexible and $\left(x^{2}, y, x\right)=0$.

1. The associative case. Let $R$ be an arbitrary nonassociative ring. The nucleus $N(R)$ of $R$ is defined by:

$$
N(R)=\{x \in R \mid(x, y, z)=(y, z, x)=(y, x, z)=0 \quad \forall y, z \in R\} .
$$

It is well known $[9$, p. 13] that $N(R)$ is an associative subring of $R$.

A ring $R$ is said to have a Peirce decomposition relative to the idempotent $e \in R$ if $R$ can be decomposed into a direct sum of the $Z$ modules $R_{i j}(i, j=0,1)$ where $R_{i j}=\{x \in R \mid x e=j x$ and $e x=i x\}$. It is known that if $R$ is an associative ring and if $e$ is an idempotent in $R$ then $R$ has a Peirce decomposition relative to $e$. Also, if $R$ has an identity element 1 and if we write $e_{1}=e$ and $e_{0}=1-e$ then $R_{i j}=e_{i} R e_{j}$ [3].

Lemma 1. Let $e$ be an idempotent of the ring $R$. Then $e \in N(R)$ if and only if $R$ has a Peirce decomposition $R=\bigoplus R_{i j}(i, j=0,1)$ relative to $e$ satisfying the property $R_{i j} R_{k l} \subseteq \delta_{j k} R_{i l}$ for $i, j, k, l=0,1$ ( $\delta$ denotes the Kronecker delta).

Proof. Let $e \in N(R)$. Imbed $R$ into the ring $R^{\prime}=Z+R$ which contains an identity element 1. Clearly $e$ and $1-e$ are in $N\left(R^{\prime}\right)$. From our earlier remark it follows that $R_{i j}=e_{i} R e_{j}$ for $i, j=0,1$. Thus $R_{i j} R_{k l}=$ $\left(e_{i} R e_{j}\right)\left(e_{k} R e_{l}\right)=e_{i} R\left(e_{j} e_{k}\right) R e_{l} \subseteq \delta_{j k} e_{i} R e_{l}=\delta_{j k} R_{i l}$

Conversely, if $R=\bigoplus_{i, j=0} R_{i j}$ such that $R_{i j} R_{k l} \subseteq \delta_{j k} R_{i l}$ and $a, b \in R$ then $a=\Sigma_{i, j=0}^{1} a_{i j}, b=\Sigma_{i, j=0}^{1} b_{i j}$. Then $(a, e, b)=\Sigma_{i, j, k, l=0}^{1}\left(a_{i j}, e, b_{k l}\right)=$ $\Sigma_{i, j, k, l=0}^{1}(j-k) a_{i j} b_{k l}=0$. Similarly $(a, b, e)=(e, a, b)=0$. Thus, $e \in N(R)$.

If a ring $R$ contains an idempotent $\neq 0,1$ and if all the idempotents of $R$ lie in $N(R)$ then we shall call $R$ a nuclear ring.

Theorem 1. A prime nuclear ring is associative.

Proof. Let $R$ be a prime nuclear ring with $e \neq 0,1$ an idempotent of $R$. By Lemma 1 we have a decomposition $R=\bigoplus R_{i j}, i, j=0,1$, relative to $e$ with $R_{i j} R_{k l} \subseteq \delta_{j k} R_{i l}$. Therefore if $i \neq j$ then $R_{i j}^{2}=0$. Thus, for $i \neq j$, $a_{i j}^{2}=0$ so that $e+a_{i j}$ is an idempotent of $R$. Since $R$ is nuclear $e+a_{i j} \epsilon$ $N(R)$. But $e \in N(R)$. Therefore $a_{i j} \in N(R)$. Thus $R_{10}+R_{01} \subseteq N(R)$. Since $N(R)$ is a subring of $R$ it follows that $R_{10} R_{01}+R_{01} R_{10} \subseteq N(R)$. This, together with the property $R_{i j} R_{k l} \subseteq \delta_{j k} R_{i l}$, allows us to conclude that 
$B=R_{10} R_{01}+R_{10}+R_{01}+R_{01} R_{10}$ is an ideal of $R$ contained in $N(R)$. Let $U=\{x \in R \mid x B=0\}$. Since $B \subseteq N(R)$ it follows that $U$ is an ideal of $R$.

Since $R$ is a prime ring $U B=0$ implies $U=0$ or $B=0$. But $B=0$ implies that $R=R_{11} \oplus R_{00}$. Thus, $R_{11}$ and $R_{00}$ are ideals of $R$ such that $R_{11} R_{00}=$ 0 . From the primeness of $R$ again $R_{11}=0$ or $R_{00}=0$. But $e \in R_{11}$ so that $R_{11} \neq 0$. Also $R_{00}=0$ implies that $e$ is the identity of $R$ contrary to hypothesis. Thus, $B \neq 0$ and $U=0$. Now, let $r_{1}, r_{2}, r_{3} \in R$ and $b \in B$. Then, since $b \in N(R),\left(r_{1}, r_{2}, r_{3}\right) b=\left(r_{1}, r_{2}, r_{3} b\right) \in(R, R, B) \subseteq(R, R, N(R))=0$. Therefore, $(R, R, R) \subseteq U=0$ so that $R$ is an associative ring.

2. The alternative case. Following $A$. Thedy [10] we define the alternative nucleus $N_{A}(R)$ of an arbitrary ring $R$ by:

$$
N_{A}(R)=\{r \in R \mid(x, r, x)=0 \text { and }(r, y, x)=(y, x, r)=(x, r, y) \quad \forall x, y \in R\} .
$$

If $R$ is 3-torsion free (i.e. if $3 a=0$ for $a \in R$ then $a=0$ ) then Thedy has shown that $N_{A}(R)$ is a subring of $R$.

Lemma 2. Let e be an idempotent of a ring $R$. Then $e \in N_{A}(R)$ if and only if $R$ has a Peirce decomposition relative to $e$ satisfying the properties:

(a) $R_{i j} R_{j k} \subseteq R_{i k}$.

(b) $R_{i j} R_{i j} \subseteq R_{j i}$

(c) $R_{i j} R_{k l}=0$ if $j \neq k$ and $(i, j) \neq(k, l)$.

(d) $r_{i j}^{2}=0$ for any $r_{i j} \in R_{i j}$, $i \neq j$.

Proof. Let $e$ be an idempotent in $N_{A}(R)$. Then from the definition of $N_{A}(R)$ one obtains as in [9, p. 33]

$$
e\left(a_{i j} b_{k l}\right)=(i+j-k) a_{i j} b_{k l}
$$

and

$$
\left(a_{i j} b_{k l}\right) e=(k+l-j) a_{i j} b_{k l}
$$

Thus (a) and (b) follow immediately. Also (d) follows from $\left(r_{i j}, r_{i j}, e\right)=0$ and property (b). To obtain (c) first note that if $x \in R$ such that $x e=s x$ for some $s \in Z$ (ex =tx for some $t \in Z$ ) then $s=0$ or $s=1 \quad(t=0$ or $t=1$ ). Now in (1) and (2) let $j=1$ and $k=0$. Then $e\left(a_{i 1} b_{0 l}\right)=(i+1) a_{i 1} b_{0 l}$ and $\left(a_{i 1} b_{0 l}\right) e=(l-1) a_{i 1} b_{0 l}$. By the preceding remark it follows that $i=0$ and $l=1$. Therefore if $a_{i 1} b_{0 l} \neq 0$ then $(i, j)=(k, l)=(0,1)$ contrary to hypothesis. The same argument applies if $j=0$ and $k=1$. 
Conversely, if $R$ has a Peirce decomposition relative to $e$ satisfying (a)-(d) then it is straightforward, using the linearity of the associator, to show that $(x, e, x)=0$ and $(x, e, y)=(y, x, e)=(e, y, x)$ for arbitrary $x, y$ in $R$. Thus, $e \in N_{A}(R)$.

We call a ring $R$ an $A$-nuclear ring if $R$ contains an idempotent $e \neq 0,1$ and if every idempotent of $R$ lies in $N_{A}(R)$.

Henceforth, assume that $R$ is an $A$-nuclear ring, $e$ an idempotent of $R$, and $R=R_{11}+R_{10}+R_{01}+R_{00}$ the Peirce decomposition relative to $e$.

Lemma 3. The set $B=R_{10} R_{01}+R_{10}+R_{01}+R_{01} R_{10}$ is an ideal of $R$.

Proof. By Lemma 2 it is sufficient to show that $R_{i i} B+B R_{i i} \subseteq B$ for $i=0,1$ which reduces to $R_{i i}\left(R_{i j} R_{j i}\right)+\left(R_{i j} R_{j i}\right) R_{i i} \subseteq B$ for $i \neq j$. Now by (d) of Lemma $2 a_{i j}^{2}=0$ for $a_{i j} \in R_{i j}, i \neq j$. Therefore $e+a_{i j}$ is an idempotent. Hence $a_{i j} \in N_{A}(R)$ if $i \neq j$ so that $\left(a_{i i}, a_{i j}, a_{j i}\right)=-\left(a_{i i}, a_{j i}, a_{i j}\right)$. Since $i \neq j$ the right-hand side is 0 and we have $\left(a_{i i} a_{i j}\right) a_{j i}=a_{i i}\left(a_{i j} a_{j i}\right)$. Thus, $R_{i i}\left(R_{i j} R_{j i}\right)=\left(R_{i i} R_{i j}\right) R_{j i} \subseteq R_{i j} R_{j i} \subseteq B$. Similarly, $\left(R_{i j} R_{j i}\right) R_{i i} \subseteq B$ so that $B$ is an ideal of $R$.

Define $U_{i}=\left\{x \in R_{i i} \mid x\left(R_{10}+R_{01}\right)=\left(R_{10}+R_{01}\right) x=0\right\}$ for $i=0,1$. Then we have:

Lemma 4. $U_{i}(i=0,1)$ is an ideal of $R$.

Proof. We prove the lemma for $U_{1}$ and note that the same proof applies for $U_{0}$. Clearly $U_{1}$ is an abelian group under addition. Let $u \in U_{1}$, $a \in R_{10}+R_{01}$, and $r \in R$. Without loss of generality we may assume that $r \in R_{11}$. Also $a \in N_{A}(R)$. Therefore $(u r) a=u(r a)-(u, a, r)$. Now $r a \in R_{10}$ and $a r \in R_{01}$. Therefore $u(r a)=0=u(a r)$. Hence $(u r) a=0$. Similarly $a(u r)=(a u) r+(u, a, r)$ so that $a(u r)=0$. Therefore $u r \in U_{1}$. In the same vein $r u \in U_{1}$. Thus $U_{1}$ is an ideal of $R$.

Lemma 5. $U_{i} B=B U_{i}=0$.

Proof. We again prove the lemma for $U_{1}$. Clearly $U_{1}\left(R_{10}+R_{01}+R_{01} R_{10}\right)=$ $\left(R_{10}+R_{01}+R_{01} R_{10}\right) U_{1}=0$ by Lemma 2 and the definition of $U_{1}$. Let $u \in U_{1}, a_{10} \in R_{10}$, and $a_{01} \in R_{01}$. Then since $a_{10} \in N_{A}(R), u\left(a_{10} a_{01}\right)=$ $\left(u a_{10}\right) a_{01}+\left(a_{10}, u, a_{01}\right)$. But since $u \in U_{1}$ the right-hand side is 0 . Therefore $u\left(R_{10} R_{01}\right)=0$. Similarly $\left(R_{10} R_{01}\right) u=0$. Therefore $U_{1} B=B U_{1}=0$ 。 
Lemma 6. If $R$ is a prime $A$-nuclear ring then $R_{11}$ and $R_{00}$ are associative subrings of $R$.

Proof. Since $R$ is a prime ring, by Lemma 5 either $B=0$ or $U_{1}=U_{0}=$ 0 . But $B=0$ implies that $R=R_{11} \oplus R_{00}$. Since $e \in R_{11}, R_{11} \neq 0$. On the other hand, $R_{00}=0$ implies that $R=R_{11}$ so that $e$ is an identity element of $R$ contrary to hypothesis. Therefore $U_{1}=U_{0}=0$. Now let $x, y, z \in R_{i i}$, $r \in R_{10}+R_{01}$. Then $r \in N_{A}(R)$ and $(x, y, z) r=[(x y) z] r-[x(y z)] r=$ $(x y, z, r)-(x, y z, r)+(x y)(z r)-x[(y z) r]=(x y, z, r)-(x, y z, r)+(x, y, z r)-$ $x(y, z, r)=0$ since $r, z r \in R_{10}+R_{01} \subseteq N_{A}(R)$ and if $a, b \cdot \in R_{i i}, r \in R_{10}+$ $R_{01}$, then $(a, b, r)=(b, r, a) \in\left[R_{i i}\left(R_{10}+R_{01}\right)\right] R_{i i}+R_{i i}\left[\left(R_{10}+R_{01}\right) R_{i i}\right]=$ 0 . In the same fashion $r(x, y, z)=0$. Therefore $(x, y, z) \in U_{i}=0$. Thus, $R_{11}$ and $R_{00}$ are associative subrings of $R$.

Theorem 2. If $R$ is a prime A-nuclear ring then $R$ is alternative.

Proof. Let $x, y \in R$. Then $x=\Sigma_{i, j=0}^{1} x_{i j}$ and $y=\Sigma_{i, j=0}^{1} y_{i j}$ so that $(x, x, y)=\Sigma_{i, j=0}^{1}\left(x, x, y_{i j}\right)$. Now if $i \neq j$ then $y_{i j} \in N_{A}(R)$ so that, by the definition of $N_{A}(R),\left(x, x, y_{10}\right)=\left(x, x, y_{01}\right)=0$. Thus, $(x, x, y)$ reduces to $\Sigma_{l=0}^{1}\left(x, x, y_{l l}\right)=\Sigma_{i, j, k, r, l=0}^{1}\left(x_{i j}, x_{k r}, y_{l l}\right)$. Let $S$ denote the sum $\Sigma_{i, j, k, r, l=0}^{1}\left(x_{i j}, x_{k r}, y_{l l}\right)$. The terms in $S$ of the form $\left(x_{j j}, x_{k k}, y_{l l}\right)$ are all zero by Lemmas 2 and 6 . The terms in $S$ of the form $\left(x_{i j}, x_{i j}, y_{l l}\right)$ for $i \neq j$ are all zero since $x_{i j} \in N_{A}(R)$. Finally, the other terms in $S$ come in pairs of the form $\left(x_{i j}, x_{k r}, y_{l l}\right)+\left(x_{k r}, x_{i j}, y_{l l}\right)$. Since $i \neq j$ or $k \neq r$ the sum of each of these pairs is zero. Thus $S=0$ so that $(x, x, y)=0$. Similarly $(y, x, x)=0$. Thus $R$ is alternative.

It is worthwhile to note that if $R$ is 3-torsion free then Theorem 2 can be obtained more directly. For, in this case, $N_{A}(R)$ is a subring of $R$. Thus $B$ is an ideal of $R$ contained in $N_{A}(R)$. Then by Lemma 3 of [10] $(x, x, y) \in B^{\perp}$ and $(y, x, x) \in B^{\perp}$ where $B^{\perp}=\{r \in R \mid r B=B r=0\}$. Since $B^{\perp}$ is an ideal of $R$ and $B B^{\perp}=B^{\perp} B=0$ while $B \neq 0$, it follows that $B^{\perp}=0$. Thus $R$ is alternative.

Theorems 1 and 2 assume that $R$ is prime and that all of the idempotents of $R$ lie in $N(R), N_{A}(R)$, respectively. The following examples show that these conditions are necessary.

Example 1. Let $F$ be a field and $R$ an algebra over $F$ with basis elements $e, b, b^{\prime}, c, f$ with multiplication given by: $e^{2}=e, f^{2}=f, e b=b f=b$, 
$e b^{\prime}=b^{\prime} f=b^{\prime}, c e=f c=c, b c=e, c b^{\prime}=f$, and all other products zero. It is straightforward to see that $e \in N(R)$ and that $R$ is a simple algebra, hence a simple ring. However, $R$ is not even alternative since $\left(b, c, b^{\prime}\right)+\left(b^{\prime}, c\right.$, $b)=b^{\prime}-b \neq 0$. This is due to the fact that $e+b$ is an idempotent of $R$ but $e+b \notin N_{A}(R)$. Note also that $R$ does not satisfy the Jordan identity $\left(x^{2}, y, x\right)=0$ since $\left(\left(f+b^{\prime}\right)^{2}, c, f+b^{\prime}\right)=-b^{\prime} \neq 0$.

Example 2. Let $R$ be a 3-dimensional algebra over a field $F$ with basis $e, a, b$ and multiplication given by: $e^{2}=e, a b=a-b, b a=b$, and all other products zero. Then $e \in N(R)$ and $e$ is the only idempotent of $R$. Thus, $R$ is a nuclear ring. In addition, $R$ is a semiprime ring. However, $R$ is not a prime ring since the ideals $\mathrm{Fe}$ and $\mathrm{Fa}+\mathrm{Fb}$ are orthogonal. $R$ is not alternative since $(a, b, b)=a-b \neq 0$. Thus, the assumption that $R$ is prime is necessary. Here again, $R$ does not satisfy $\left(x^{2}, y, x\right)=0$.

3. The Jordan case. Henceforth we must assume that all of our rings $R$ satisfy the condition that to each $a \in R$ there exists a unique $b \in R$ such that $2 b=a$. We write $b=1 / 2 a$. It is known [2], [4] that if $R$ is a Jordan ring and if $e$ is an idempotent of $R$ then $R$ has a decomposition $R=R_{1}+R_{1 / 2}+$ $R_{0}$ where $R_{i}=\{x \in R \mid x e=e x=i x\}$. Also, the modules $R_{i}$ satisfy the multiplicative properties:

(i) $R_{i}^{2} \subseteq R_{i}$ for $i=0,1 ; R_{1 / 2}^{2} \subseteq R_{1}+R_{0}, R_{1} R_{0}=0, R_{i} R_{1 / 2} \subseteq R_{1 / 2}$ for $i=$ 0,1 .

Thus, if $a, b \in R_{1 / 2}$ then $a b \in R_{1}+R_{0}$. We denote this by $a b=(a b)_{1}+$ $(a b)_{0}$. It is also known that products of elements of the different $R_{i}$ satisfy:

(ii) (a) $x_{1 / 2}\left(y_{i} z_{i}\right)=\left(x_{1 / 2} y_{i}\right) z_{i}+\left(x_{1 / 2} z_{i}\right) y_{i}, i=0,1$.

(b) $x_{i}\left(y_{1 / 2} z_{1 / 2}\right)=\left[\left(x_{i} y_{1 / 2}\right) z_{1 / 2}+\left(x_{i} z_{1 / 2}\right) y_{1 / 2}\right]_{i}, i=0,1$.

(c) $\left[\left(x_{1} y_{1 / 2}\right) z_{1 / 2}\right]_{0}=\left[\left(x_{1} z_{1 / 2}\right) y_{1 / 2}\right]_{0}$.

(d) $\left[\left(x_{0} y_{1 / 2}\right) z_{1 / 2}\right]_{1}=\left[\left(x_{0} z_{1 / 2}\right) y_{1 / 2}\right]_{1}$.

(e) $\left(x_{1} y_{1 / 2}\right) z_{0}=x_{1}\left(y_{1 / 2} z_{0}\right)$.

We define the Jordan nucleus, $N_{J}(R)$, of a commutative ring $R$ by:

$$
\begin{aligned}
N_{J}(R)=\{a \in R \mid(a b)(c d) & +(a d)(b c)+(a c)(b d)=[b(c d)] a+[b(a c)] d+[b(a d)] c \\
& =[a(b c)] d+[a(b d)] c+[a(c d)] b \text { for all } b, c, d \in R\} .
\end{aligned}
$$

Thus, an element $a \in R$ is in $N_{J}(R)$ if it satisfies the linearized version of the Jordan identity.

Lemma 7. Let $e$ be an idempotent of a commutative ring $R$. Then $e \in$ $N_{J}(R)$ if and only if the elements of the spaces $R_{i}$ relative to $e$ satisfy (i) and (ii). 
Proof. (i) and (ii) are established for Jordan rings in [4]. Since the procedure in all cases is to linearize the Jordan identity and to specialize by setting one of the elements equal to $e$ we may conclude immediately that $e \in N_{J}(R)$ implies (i) and (ii).

One may verify directly that if (i) and (ii) are satisfied then $e \in N_{J}(R)$ by setting $a=e$ in the definition of $N_{J}(R)$ and decomposing $b, c$ and $d$ into their components. The proof is straightforward but the computations are lengthy. We do not present the computations here.

If $R$ is a commutative ring with at least one idempotent $e \neq 0,1$ lying in $N_{J}(R)$ then we call $R$ a $J$-nuclear ring. Osborn has shown [6], [7, Proposition 6.7] that if $R$ is a commutative ring satisfying (i) and (ii) then $R$ is a Jordan ring if and only if $R_{1}$ and $R_{0}$ are Jordan rings. Thus if $R$ is simple then $R$ is Jordan. The following theorem draws from and generalizes Osborn's result.

Theorem 3. If $R$ is a prime J-nuclear ring then $R$ is a Jordan ring.

Proof. Let $e$ be an idempotent $\neq 0,1$ in $R$ such that $e \in N_{J}(R)$. By Lemma 7 we have (i) and (ii). Let $A=\left(R_{1 / 2} R_{1 / 2}\right)_{1}+R_{1 / 2}+\left(R_{1 / 2} R_{1 / 2}\right)_{0}$. It follows from (i) and (ii) (b) that $A$ is an ideal of $R$. Also, let $C_{i}=\{x \in$ $\left.R_{i} \mid x R_{1 / 2}=0\right\}$ for $i=0,1$. It follows from (i) and (ii) (a) that $C_{i}, i=0,1$, is an ideal of $R$. Also, from (i) and (ii) (b) $A C_{1}=A C_{0}=0$. Since $R$ is a prime ring either $A=0$ or $C_{1}=C_{0}=0$. But $A=0$ implies that $R=R_{1} \oplus R_{0}$. This, however, is impossible as in Theorem 2. Therefore $C_{1}=C_{0}=0$.

From (ii) (a) we have a homomorphism $\phi_{i}$ from $R_{i}$ into $\operatorname{Hom}\left(R_{1 / 2}, R_{1 / 2}\right)^{+}$ with $\operatorname{Ker} \phi_{i}=C_{i}$ for $i=0,1$ [2]. Since $C_{1}=C_{0}=0$ we have $R_{1}$ and $R_{0}$ imbedded in the Jordan ring $\operatorname{Hom}\left(R_{1 / 2}, R_{1 / 2}\right)^{+}$. Therefore $R_{1}$ and $R_{0}$ are Jordan rings and by [7, Proposition 6.7] it follows that $R$ is a Jordan ring.

4. The noncommutative Jordan case. Recall that a ring $R$ is a noncommutative Jordan ring if it is flexible and satisfies the identity $\left(x^{2}, y\right.$, $x)=0$. It is known [1], [5] that if $e$ is an idempotent of a noncommutative Jordan ring $R$ then $R$ has a decomposition $R=R_{1}+R_{1 / 2}+R_{0}$ relative to $e$ where $R_{i}=\{x \in R \mid x e=e x=i x\}$ for $i=0,1$ and $R_{1 / 2}=\{x \in R \mid x e+e x=x\}$. Multiplication among the $R_{i}$ is given by:

(iii) $R_{i}^{2} \subseteq R_{i}, R_{i} R_{1 / 2}+R_{1 / 2} R_{i} \subseteq R_{1 / 2}, i=0,1, R_{1} R_{0}=R_{0} R_{1}=0$ and if $x, y \in R_{1 / 2}$ then $x y+y x \in R_{1}+R_{0}$.

Assume now that $R$ is a flexible ring in which for every $a$ in $R$ there is a unique $b$ in $R$ such that $2 b=a$. We define the noncommutative Jordan 
nucleus, $N_{N J}(R)$, of $R$ by:

$$
\begin{array}{r}
N_{N J}(R)=\left\{a \in R \mid\left[E_{a x+x a}, F_{z}\right]+\left[E_{a z+z a}, F_{x}\right]+\left[E_{x z+z x}, F_{a}\right]=0\right. \\
=a\left(\left[E_{x y+y x}, F_{z}\right]+\left[E_{x z+z x}, F_{y}\right]+\left[E_{z y+y z}, F_{x}\right]\right)
\end{array}
$$

for all $x, y, z$ in $R\}$

where $E, F=r, l$ and $r_{x}\left(l_{x}\right)$ denotes right (left) multiplication by the element $x$. It is a straightforward matter to show that $N_{N J}(R) \subseteq N_{J}\left(R^{+}\right)$. The properties (iii) are obtained for noncommutative Jordan rings by linearizing the Jordan identities and setting one of the variables equal to $e$. McCrimmon [5] has shown by the same method that

$$
e(z y+y z)=z y, \quad(y z+z y) e=y z \quad \text { if } y \in R_{0} \text { and } z \in R_{1 / 2}
$$

and

$$
z l_{x y}=z l_{y} l_{x}+z r_{x} l_{y} \text { and } z r_{x y}=z l_{y} r_{x}+z r_{x} r_{y}
$$

$$
\text { if } x, y \in R_{0} \text { and } z \in R_{1 / 2} \text {. }
$$

If $R$ contains an idempotent $e \neq 0,1$ such that $e \in N_{N J}(R)$ then we shall call $R$ an $N J$-nuclear ring. Thus, in an $N J$-nuclear ring (iii), (3) and (4) hold. Similarly, we have

$$
e(z y+y z)=y z, \quad(y z+z y) e=z y \quad \text { if } y \in R_{1} \text { and } z \in R_{1 / 2}
$$

and

$$
z l_{x y}=z l_{y} l_{x}+z l_{x} r_{y}, \quad z r_{x y}=z r_{x}^{r} y+z r_{y} l_{x}
$$

$$
\text { if } x, y \in R_{1} \text { and } z \in R_{1 / 2} \text {. }
$$

For, since $R$ is flexible, $l_{a y}-l_{y} l_{a}=r_{y a}-r_{y} r_{a}$ for all $a, b \in R$. In particular, if $a=e, y \in R_{1}$ and we allow this to act on $z \in R_{1 / 2}$ we get $y z-$ $e(y z)=z y-(z y) e$ or $y z-z y=e(y z)-(z y) e$. Add and subtract $e(z y)$ to the right side of this equation to get $y z-z y=e(y z)+e(z y)-z y$. Therefore $y z=e(y z)+e(z y)$. The second half of $\left(3^{\prime}\right)$ follows in a similar manner.

For the first half of $\left(4^{\prime}\right)$ let $E=l, F=r, a=e, x \in R_{1}$, and $z \in R_{1 / 2}$ in the definition of $N_{N J}(R)$ to get $2\left[l_{x}, r_{z}\right]+\left[l_{z}, r_{x}\right]+\left[l_{x z+z x}, r_{e}\right]=0$ which, by flexibility, reduces to $\left[l_{x}, r_{z}\right]+\left[l_{x z+z x}, r_{e}\right]=0$. If we allow this to act on $y \in R_{1}$ we get $(x y) z-x(y z)+[(x z+z x) y] e-(x z+z x) y=0$. Again, in the definition of $N_{N J}(R)$ let $x \in R_{1}, a=z=e$ to obtain $2\left[E_{x}, F_{e}\right]+$ $2\left[E_{e}, F_{x}\right]+2\left[E_{x}, F_{e}\right]=0$. By flexibility $\left[E_{x}, F_{e}\right]+\left[E_{e}, F_{x}\right]=0$. There- 
fore, we have

$$
\left[E_{x}, F_{e}\right]=0 \text { if } x \in R_{1} \text {. }
$$

Therefore $[(x z+z x) y] e=[(x z+z x) e] y=(z x) y$ by $\left(3^{\prime}\right)$. Thus, we now have $(x y) z-x(y z)-(x z) y=0$ which reduces to $z l_{x y}=z l_{y} l_{x}+z l_{x}{ }^{r} y^{\bullet}$ Similarly if we let $E=r, F=l, x \in R_{1}, z \in R_{1 / 2}$, and $a=e$ we get the second half of $\left(4^{\prime}\right)$.

Lemma 8. Let $R$ be an $N\}$-nuclear ring with $K_{i}=\left\{x \in R_{i} \mid x R_{1 / 2}=R_{1 / 2} x=\right.$ 0\} for $i=0,1$. Then $K_{i}$ is an ideal of $R$.

Proof. If $i=0$ this follows from (iii) and (4) while if $i=1$ it follows from (iii) and $\left(4^{\prime}\right)$.

Lemma 9. If $R$ is an $N J$-nuclear ring and $C_{i}=\left\{x \in R_{i} \mid x \cdot R_{1 / 2}=0\right\}, i=$ 0,1 then $K_{i}=C_{i}$.

Proof. Clearly $K_{i} \subseteq C_{i}$. Let $y \in C_{i}, z \in R_{1 / 2}$. Then $y z+z y=0$. Then if $i=0$, (3) gives $y z=z y=0$; whereas, if $i=1$, one gets the same result from ( $\left.3^{\prime}\right)$. Thus, $y \in K_{i}$.

We have noted earlier that if $x \in R_{1}$ and $z \in R_{1 / 2}$ in an $N J$-nuclear ring then $\left[l_{x}, r_{z}\right]+\left[l_{x z+z x}, r_{e}\right]=0$. From flexibility we also get $\left[l_{z}, r_{x}\right]+$ $\left[l_{e}, r_{x z+z x}\right]=0$. If we allow these to act on $y \in R_{1 / 2}$ we obtain:

$$
(x y) z-x(y z)+[(x z+z x) y] e-(x z+z x)(y e)=0
$$

and

$$
\begin{aligned}
(z y) x-z(y x)+(e y)(x z+z x)-e[y(x z+z x)] & =0, \\
& \text { if } y, z \in R_{1 / 2} \text { and } x \in R_{1} .
\end{aligned}
$$

Similarly, if $x \in R_{0}, z \in R_{1 / 2}$ and $a=e$ in the definition of $N_{N J}(R)$ we get $\left[E_{z}, F_{x}\right]+\left[E_{x z+z x}, F_{e}\right]=0$. If we allow this to act on $y \in R_{1 / 2}$ we obtain:

$$
x(y z)-(x y) z+e[y(x z+z x)]-(e y)(x z+z x)=0
$$

and

$\left(7^{\prime}\right)$

$$
(z y) x-z(y x)+[(x z+z x) y] e-(x z+z x)(y e)=0,
$$

$$
\text { if } y, z \in R_{1 / 2} \text { and } x \in R_{0} \text {. }
$$

We are now able to prove:

Theorem 4. A prime NJ-nuclear ring is a noncommutative Jordan ring.

Proof. We first show that $K_{0}=K_{1}=0$. As in Theorem 3 let $A=$ 
$\left(R_{1 / 2} R_{1 / 2}\right)_{0}+R_{1 / 2}+\left(R_{1 / 2} R_{1 / 2}\right)_{1}$. Then as in [5, Lemma 2] $A$ is an ideal of $R$. Now by (iii), (6), and (7), $A K_{1}=K_{1} A=0$; whereas by (iii), ( $\left.6^{\prime}\right)$, and ( $\left.7^{\prime}\right)$, $A K_{0}=K_{0} A=0$. Now, if $A=0$ then $R_{1 / 2}=0$ which is impossible since $R$ is a prime ring. Therefore $K_{1}=K_{0}=0$. Thus, by Lemma 9, $C_{1}=C_{0}=0$. Therefore $R^{+}$is a J-nuclear ring in which $C_{1}=C_{0}=0$. As in Theorem 3 it follows that $R_{1}^{+}$and $R_{0}^{+}$are Jordan rings. Therefore by [6], [7], $R^{+}$is a Jordan ring. Since $R$ is flexible and $R^{+}$is Jordan, it follows [8] that $R$ is a noncommutative Jordan ring.

Finally, note that our Example 1 earlier shows that it is not true that in a prime nonflexible ring $e \in N_{N J}(R)$ implies that $R=N_{N J}(R)$.

\section{REFERENCES}

1. A. A. Albert, Power-associative rings, Trans. Amer. Math. Soc. 64 (1948), 552-593. MR 10, 349.

2. - A theory of powerassociative commutative algebras, Trans. Amer. Math. Soc. 69 (1950), 503-527. MR 12, 475.

3. N. Jacobson, Structure of rings, 2nd rev. ed., Amer. Math. Soc. Colloq. Publ., vol. 37, Amer. Math. Soc., Providence, R. J., 196́4. MR 36 \#5158.

4. - Structure and representations of Jordan algebras, Amer. Math. Soc. Colloq. Publ., vol. 39, Amer. Math. Șoc., Providence, R. Io, 1968. MR 40 \#4330.

5. K. McCrimmon, Structure and representations of noncommutative Jordan algebras, Trans. Amer. Math. Soc. 121 (1966), 187-199. MR 32 \#5700.

6. J. M. Osborn, Commutative algebras satisfying an identity of degree four, Proc. Amer. Math. Soc. 16 (1965), 1114-1120. MR 32 \#2451.

7. - Varieties of algebras, Advances in Math. 8 (1972), 163-369. MR $44 \# 67 \div 5$.

8. R. D. Schafer, Noncommutative Jordan algebras of characteristic 0, Proc. Amer. Math. Soc. 6 (1955), 472-475. MR 17, 10.

9. - An introduction to nonassociative algebras, Pure and Appl. Math., vol. 22, Academic Press, New York and London, 1966. MR 35 \#1643.

10. A. Thedy, On rings with completely alternative commutators, Amer. J. Math. 93 (1971), 42-51. MR $43 \# 300$.

DEPARTMENT OF MATHEMATICS, TEMPLE UNIVERSITY, PHILADELPHIA, FENNSYLVANIA 19122 